\title{
Indirect retention
}

\author{
J. C. Davenport, ' R. M. Basker, ${ }^{2}$ J. R. Heath, ${ }^{3}$ J. P. Ralph, ${ }^{4}$ \\ P-O. Glantz, ${ }^{5}$ and P. Hammond, ${ }^{6}$
}

\section{This article explains the mechanism of indirect retention for RPDs and discusses the factors which determine its effectiveness. Examples are given of designs which incorporate indirect retention.}

The principle of indirect retention may be explained by 1 reference to the behaviour of a mandibular distal extension saddle in function.
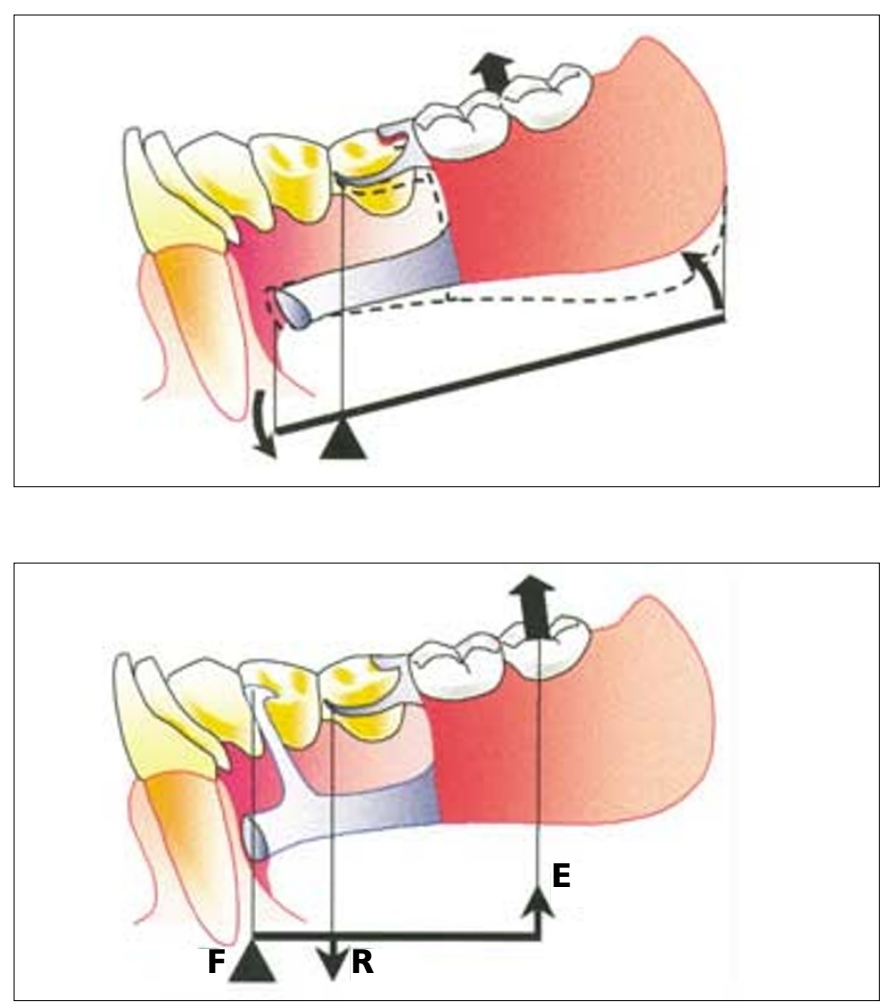

\footnotetext{
$1^{*}$ Emeritus Professor, University of Birmingham, UK; ${ }^{2}$ Professor of Dental Prosthetics, University of Leeds and Consultant in Restorative Dentistry, Leeds Teaching Hospitals NHS Trust, Leeds, UK; ${ }^{3}$ Honorary Research Fellow, University of Manchester (Formerly Senior Lecturer in Restorative Dentistry, University of Manchester) and Consultant in Restorative Dentistry, Central Manchester Healthcare Trust, Manchester, UK; ${ }^{4}$ Consultant in Restorative Dentistry, Leeds Teaching Hospitals NHS Trust and Senior Clinical Lecturer, University of Leeds and Honorary Visiting Professor, Centre for Dental Services Studies, University of York, York, UK; ${ }^{5}$ Professor of Prosthetic Dentistry, Consultant in Prosthetic Dentistry, Faculty of Odontology, University of Malmo, Sweden; ${ }^{6}$ Professor of Informatics, Eastman Dental Institute for Oral Health Care Sciences, University College London ${ }^{*}$ Correspondence to: 5 Victoria Road, Harborne, Birmingham B17 0AG email:john.davenport@btclick.com

REFEREED PAPER (C) British Dental Journal 2001; 190: 128-132
}

In this part, we will discuss

- Indirect retention systems

- Clasp axis

- Importance of clasps

- Mechanical disadvantage of RPD designs

- Support for indirect retainers

\section{Fig. I - Indirect retention}

This saddle has an occlusal rest and a clasp on the abutment tooth, and the connector is a sublingual bar. Although normally a mesial rest might well be preferred, a distal rest has been used in this example to simplify the explanation which follows. When sticky foods displace the saddle in an occlusal direction the tips of the retentive clasps engaging the undercuts on the abutment teeth provide the only mechanical resistance to the movement. The saddle thus pivots about the clasp tips.

In the maxilla this movement of the saddle away from the ridge may also be caused by gravity.

\section{Fig. 2 - Indirect retention}

If the design is modified by placing a rest on an anterior tooth, this rest (indirect retainer) becomes the fulcrum of movement of the saddle in an occlusal direction causing the clasp to move up the tooth, engage the undercut and thus resist the tendency for the denture to pivot.

$\mathrm{F}=$ Fulcrum - indirect retainer, a component which obtains support. $\mathrm{R}=$ Resistance - retention generated by the clasp.

$E=$ Effort - displacing force, eg a bolus of sticky food.

It can thus be seen that to obtain indirect retention the clasp must always be placed between the saddle and the indirect retainer. 


\section{PRACTICE prosthetics}

\section{Fig. 3 - Indirect retention}

Indirect retainers do not prevent displacement towards the ridge. This movement is resisted by the occlusal rest on the abutment tooth and by full extension of the saddle to gain maximum support from the residual ridge. In addition, it may be necessary to compensate for the compressibility of the denture-bearing mucosa by using the altered cast impression technique (A Clinical Guide to Removable Partial Dentures, Chapter 19).

\section{Fig. 4 - Indirect retention}

In order to understand the way in which indirect retainers are located it is necessary to consider the possible movement of the denture around an axis formed by the clasps. This clasp axis is defined as the line drawn between the retentive tips of a pair of clasps on opposite sides of the arch.
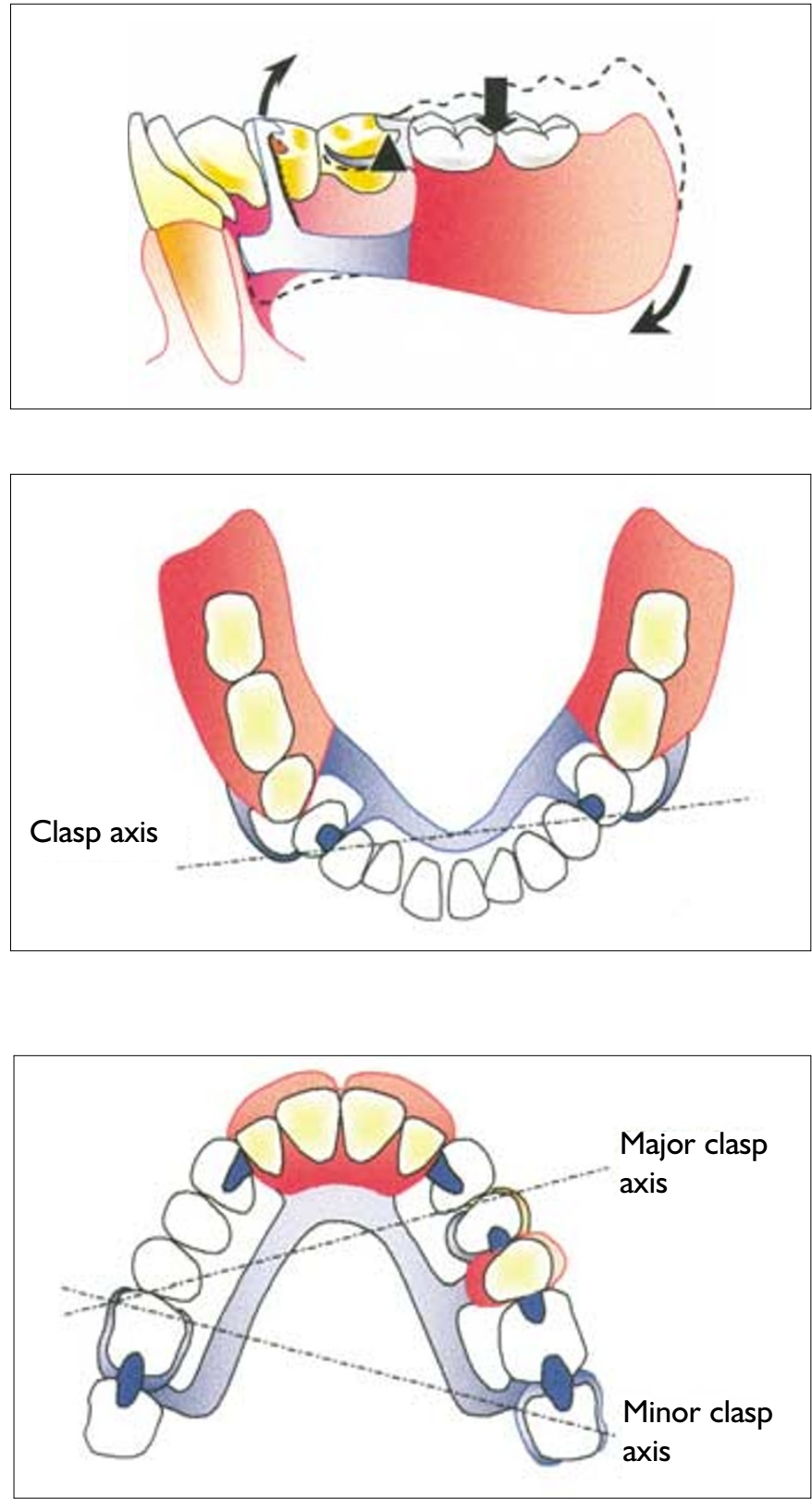

Other factors which influence the effectiveness of indirect retention are:

- The mechanical disadvantage of the denture design,

- The support of the indirect retainers.

\section{Mechanical disadvantage of the denture design}

\section{Fig. 6 - Mechanical disadvantage of the denture design}

The clasp is always nearer to the indirect retainer (fulcrum) than is the displacing force. The clasp is therefore working at a mechanical disadvantage relative to the displacing force.

The RPD design should strive to reduce the mechanical advantage of the displacing force by placing the clasp axis as close as possible to the saddle and by placing the indirect retainers as far as possible from the saddle.

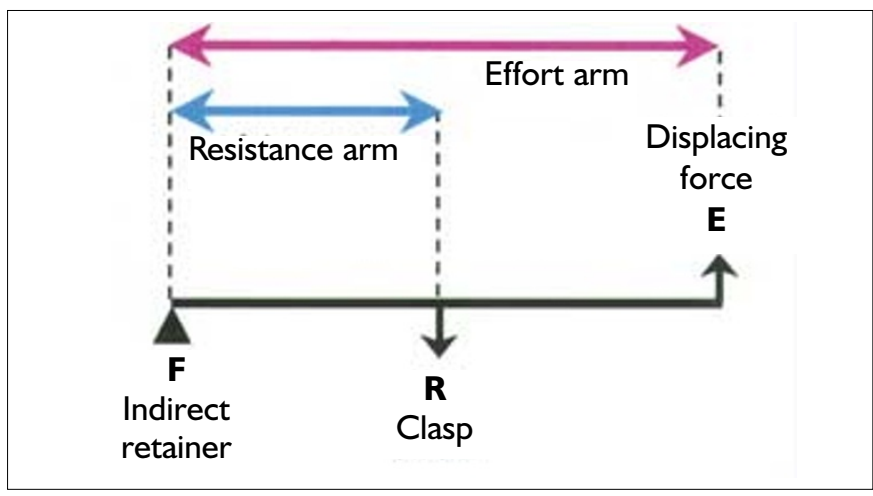



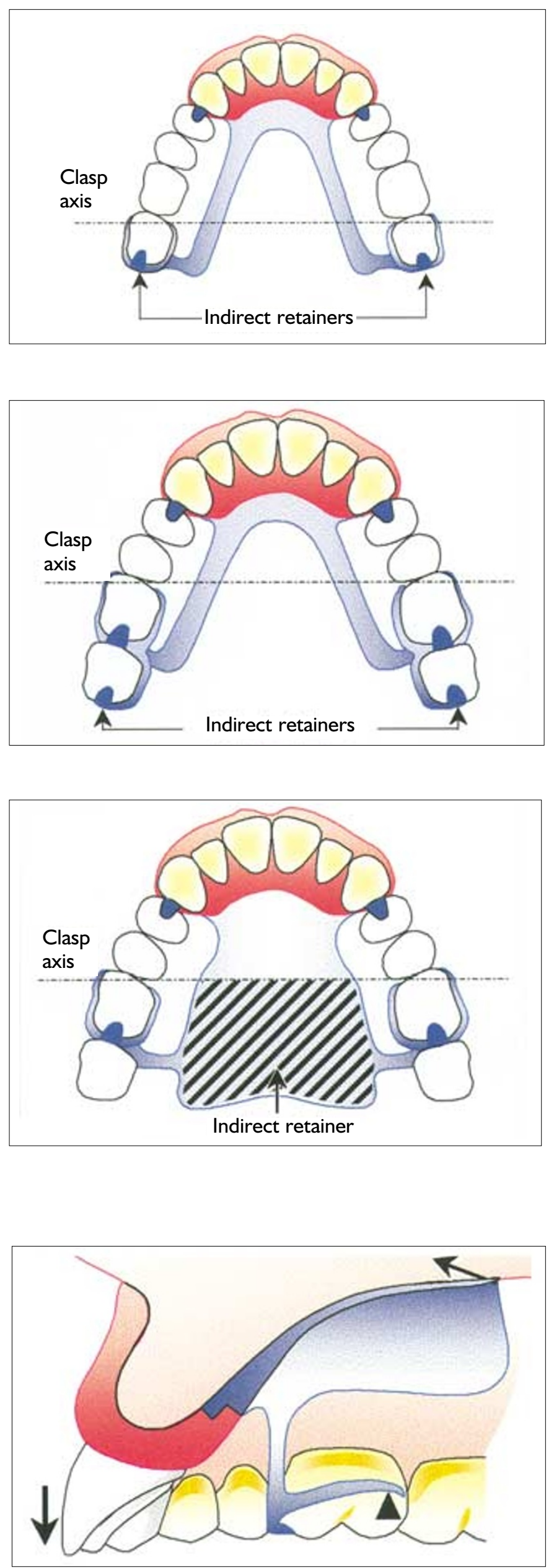

Fig. 7 - Mechanical disadvantage of the denture design

In this RPD design the indirect retainers (the rests on the molar teeth) are inefficient because they are placed too close to the clasp axis.

Fig. 8 - Mechanical disadvantage of the denture design If the clasp axis is moved closer to the saddle the effectiveness of the indirect retention is improved.

\section{Support for the indirect retainer}

Fig. 9 - Support for the indirect retainer

Tooth support is preferable to mucosal support because the compressibility of mucosa allows movement of the denture to occur.

If there is no alternative to mucosal support the indirect retainer should cover a sufficiently wide area to spread the load and avoid mucosal injury. This consideration effectively limits mucosally supported indirect retainers to the maxilla where the load can be distributed over the hard palate (shaded area of the connector). However, this plan view is somewhat misleading as it suggests that the indirect retention achieved is more effective than it really is.

Fig. 10 - Support for the indirect retainer

The side view (simplified) of a similar design shows that, when the saddle is first displaced, mucosal compression beneath the indirect retainer allows the denture to rotate around the clasp axis (fulcrum). The path of movement of the indirect retainer is thus directed obliquely, rather than at right angles, to the mucosal surface. This combination of oblique approach and mucosal compression may allow a significant degree of movement of the denture in function. 


\section{PRACTICE prosthetics}

\section{Fig. I I - Support for the indirect retainer}

(I) When possible, the indirect retainer should rest on a surface at right angles to its potential path of movement. (2) If it rests on an inclined tooth surface, movement of the tooth might occur with resulting loss of support for the indirect retainer.
I

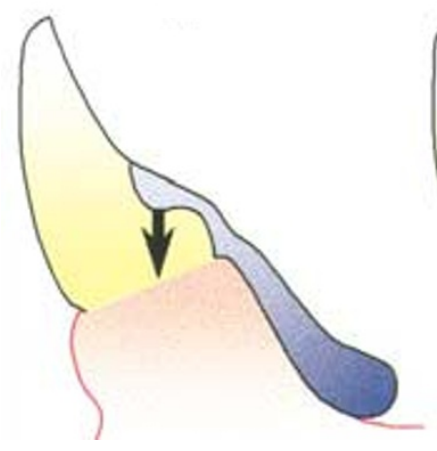

2

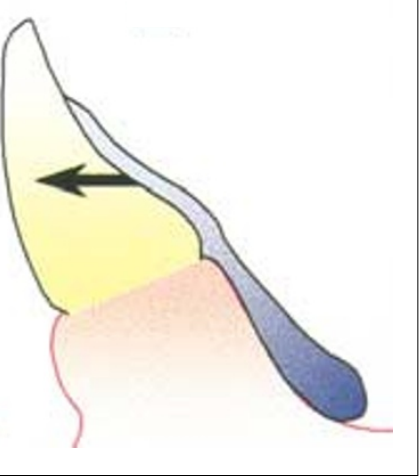

\section{Examples of RPD designs which include indi- rect retention}

Each design is only one of a number of possible solutions.

\section{Fig. $12-R P D$ designs which include indirect retention}

Kennedy I: Indirect retention in this design is provided by incisal rests on LR3 (43) and LL3 (33).

In this example and in Figs 13 to 15 the part of the saddle susceptible to displacement in an occlusal direction is indicated by an asterisk.
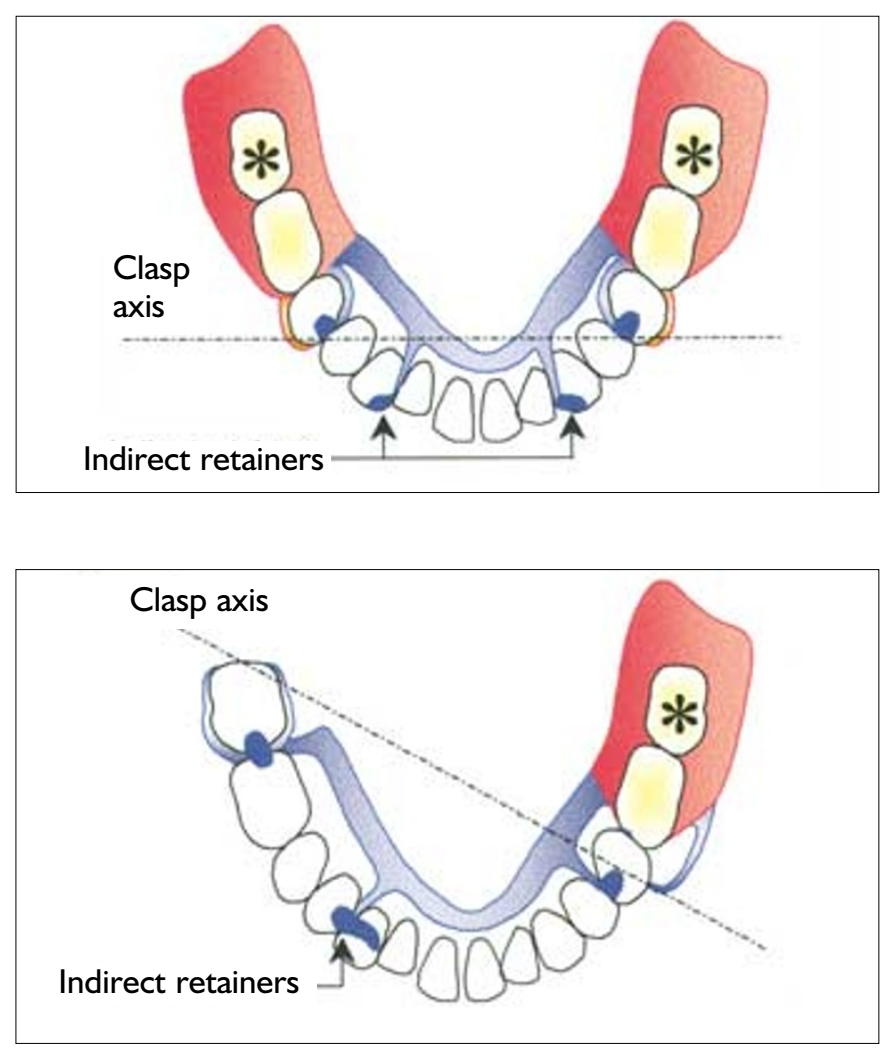

Fig. 13 - RPD designs which include indirect retention

Kennedy II: Indirect retention in this instance is provided primarily by rests on LR4 (44) and LR3 (43) as they are furthest from the clasp axis. The rests on LL5 (35), LR6 (46) and LR7 (47) are close to the clasp axis and therefore contribute little to the indirect retention.

Fig. 14 - RPD designs which include indirect retention

Kennedy III: In the case of a bounded saddle there is the potential for direct retention from both abutments. When this can be achieved, as for the saddle replacing UR6 (16) and UR5 (I5), indirect retention is not required. However, it is not uncommon for only one of the abutments to be suitable for clasping. In this design a clasp on UL3 (23) has been omitted for aesthetic reasons. Under such circumstances indirect retention can be employed, the major contribution being made by the rest on UR7 (17).

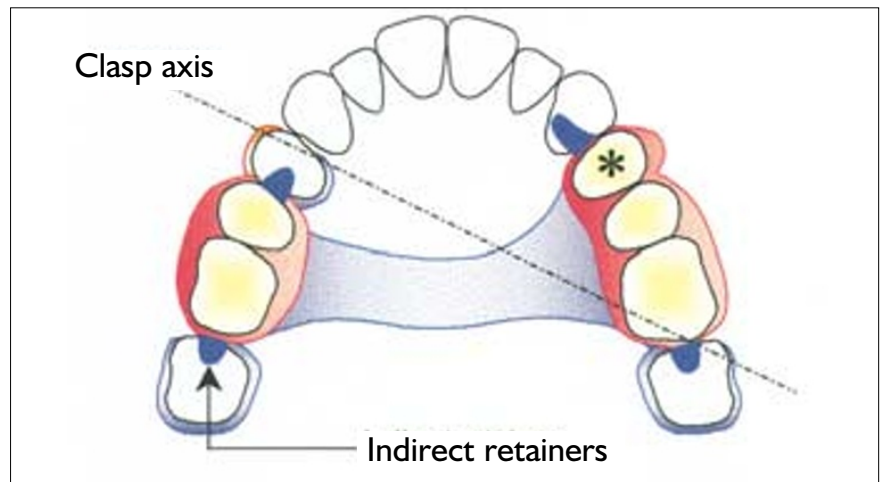




\section{prosthetics}

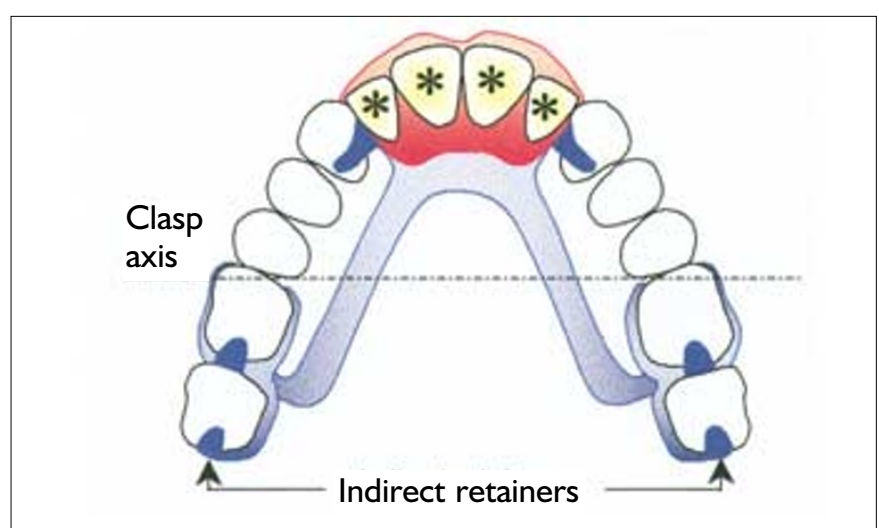

An additional function of indirect retainers is to allow accurate location of the RPD framework against the teeth when undertaking the altered cast procedure (A Clinical Guide to Removable
Fig. 15-RPD designs which include indirect retention Kennedy IV: In a maxillary denture it is sometimes difficult to achieve much separation of the clasp axis and indirect retainers. In this example, clasps engage the mesiobuccal undercuts on UR6 (16) and UL6 (26) and indirect retention has been achieved by placing the rests on UR7 (17) and UL7 (27) as far posteriorly as possible.

Partial Dentures, chapter 19), or when obtaining a wash impression to rebase a distal extension saddle. 